\title{
Review Article \\ Endogenous Sulfur Dioxide: A New Member of Gasotransmitter Family in the Cardiovascular System
}

\author{
Yaqian Huang, ${ }^{1}$ Chaoshu Tang, ${ }^{2,3} J u n b a o ~ D u,{ }^{1,3}$ and Hongfang Jin ${ }^{1}$ \\ ${ }^{1}$ Department of Pediatrics, Peking University First Hospital, Beijing 100034, China \\ ${ }^{2}$ Department of Physiology and Pathophysiology, Peking University Health Science Centre, Beijing 100191, China \\ ${ }^{3}$ Key Laboratory of Molecular Cardiology, Ministry of Education, Beijing 100191, China \\ Correspondence should be addressed to Hongfang Jin; jinhongfang51@126.com
}

Received 15 September 2015; Accepted 28 October 2015

Academic Editor: Jin-Song Bian

Copyright (C) 2016 Yaqian Huang et al. This is an open access article distributed under the Creative Commons Attribution License, which permits unrestricted use, distribution, and reproduction in any medium, provided the original work is properly cited.

\begin{abstract}
Sulfur dioxide $\left(\mathrm{SO}_{2}\right)$ was previously regarded as a toxic gas in atmospheric pollutants. But it has been found to be endogenously generated from metabolism of sulfur-containing amino acids in mammals through transamination by aspartate aminotransferase (AAT). $\mathrm{SO}_{2}$ could be produced in cardiovascular tissues catalyzed by its synthase AAT. In recent years, studies revealed that $\mathrm{SO}_{2}$ had physiological effects on the cardiovascular system, including vasorelaxation and cardiac function regulation. In addition, the pathophysiological effects of $\mathrm{SO}_{2}$ were also determined. For example, $\mathrm{SO}_{2}$ ameliorated systemic hypertension and pulmonary hypertension, prevented the development of atherosclerosis, and protected against myocardial ischemia-reperfusion (I/R) injury and isoproterenol-induced myocardial injury. These findings suggested that endogenous $\mathrm{SO}_{2}$ was a novel gasotransmitter in the cardiovascular system and provided a new therapy target for cardiovascular diseases.
\end{abstract}

\section{Introduction}

Sulfur dioxide $\left(\mathrm{SO}_{2}\right)$ was regarded as a toxic gas and environmental pollutant. It is colorless, transparent, odorous, and water-soluble. The harmful effects of $\mathrm{SO}_{2}$ on human, animals, and plants have been extensively investigated $[1,2]$. However, $\mathrm{SO}_{2}$ can be endogenously generated from metabolism of the sulfur-containing amino acid L-cysteine in mammals [3]. It has features of low molecular weight, continuous production, and fast diffusion and plays extensive biological action independent of membrane receptors $[4,5]$. In neutral fluid or mammal plasma, $\mathrm{SO}_{2}$ is broken down to its derivatives, bisulfite and sulfite $\left(\mathrm{NaHSO}_{3} / \mathrm{Na}_{2} \mathrm{SO}_{3}, 1: 3 \mathrm{M} / \mathrm{M}\right)$, maintaining organism homeostasis [6]. The sulfite is the physiological form of $\mathrm{SO}_{2}$ in vivo $[7,8]$. The reference range for total serum sulfite in healthy human beings was $0-9.85 \mu \mathrm{mol} / \mathrm{L}$ detected by high-performance liquid chromatography with fluorescence detection [9]. Serum sulfite was obviously increased in patients suffering from acute pneumonia and chronic renal failure, as well as pediatric acute lymphoblastic leukemia with bacterial inflammation [10-12]. Of note, Balazy et al. found that $\mathrm{SO}_{2}$ could be produced in the porcine coronary arterial rings after incubation with calcium ionophore by gas chromatography-mass spectrometry [13]. Du et al. firstly found that endogenous $\mathrm{SO}_{2}$ /aspartate aminotransferase (AAT) pathway existed in the cardiovascular system [14]. $\mathrm{SO}_{2}$ not only has important physiological effects on vascular tone and cardiac function but also exerts pathophysiological effects in the cardiovascular system, including regulation of hypertension, pulmonary hypertension, atherosclerosis, and cardiac ischemia-reperfusion (I/R) injury [15-18]. The abovementioned evidence suggests that the endogenous $\mathrm{SO}_{2}$ may be a novel gasotransmitter in mammals, similar to nitric oxide (NO), carbon monoxide (CO), and hydrogen sulfide $\left(\mathrm{H}_{2} \mathrm{~S}\right)$. The physiological significance of $\mathrm{SO}_{2}$, particularly its regulatory role in the cardiovascular system, has attracted a great deal of interest in the field [19-21].

Therefore, the objective of this review was to elaborate on the generation and metabolism of endogenous $\mathrm{SO}_{2}$ and give a summary of the physiological and pathophysiological effects of $\mathrm{SO}_{2}$ on the cardiovascular system. 


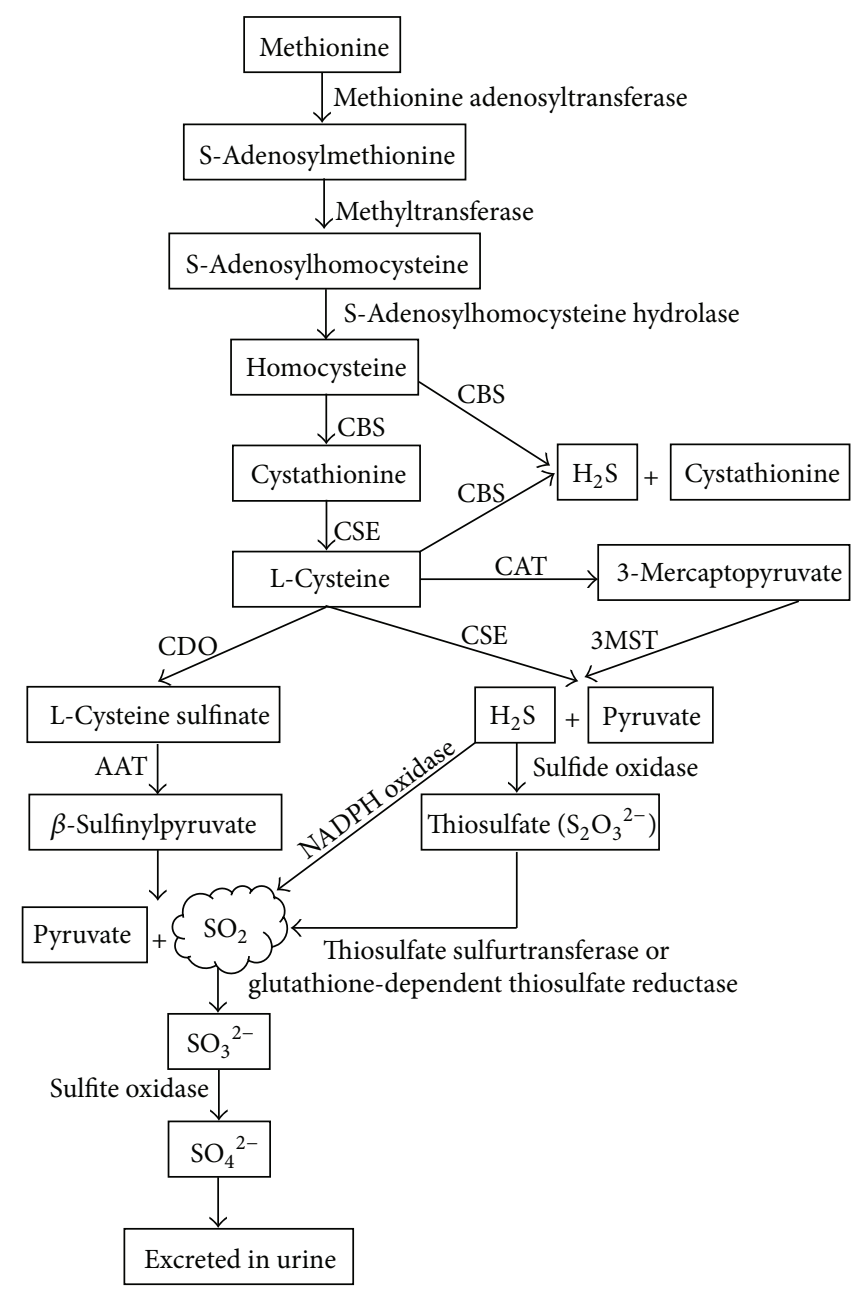

FIGURE 1: Illustration of production and metabolism of endogenous $\mathrm{SO}_{2}$ in mammals. $\mathrm{SO}_{2}$ : sulfur dioxide; $\mathrm{H}_{2} \mathrm{~S}$ : hydrogen sulfide; CBS: cystathionine $\beta$-synthase; CSE: cystathionine $\gamma$-lyase; CDO: cysteine dioxygenase; AAT: aspartate aminotransferase; CAT: 2 -cysteine aminotransferase; 3MST: 3-mercaptopyruvate sulfurtransferase.

\section{Generation and Distribution of Endogenous $\mathrm{SO}_{2}$ in the Cardiovascular System}

$\mathrm{SO}_{2}$ can be generated from the metabolism of L-cysteine which is converted from methionine via the transmethylation-transsulfuration pathway (Figure 1) [3, 22]. Firstly, Lcysteine is oxidized to form L-cysteine sulfinate by cysteine dioxygenase $(\mathrm{CDO})$, and then the latter is transaminated to form $\beta$-sulfinylpyruvate by AAT. The $\beta$-sulfinylpyruvate spontaneously decomposes to pyruvate and $\mathrm{SO}_{2}$ (Figure 1) [3]. Additionally, $\mathrm{H}_{2} \mathrm{~S}$ which shares the same substrate Lcysteine with $\mathrm{SO}_{2}$ can be transferred to $\mathrm{SO}_{2}$ in vivo through other pathways. Mitsuhashi et al. reported that $\mathrm{H}_{2} \mathrm{~S}$ could be converted to sulfite or $\mathrm{SO}_{2}$ by NADPH oxidase in activated neutrophils [23]. Besides, $\mathrm{H}_{2} \mathrm{~S}$ can be first oxidized to thiosulfate by sulfide oxidase and then converted to $\mathrm{SO}_{2}$ catalyzed by thiosulfate sulfurtransferase or glutathione-dependent thiosulfate reductase (Figure 1) $[6,24,25] . \mathrm{SO}_{2}$ can exist in the gaseous form or be hydrated to sulfite, which is subsequently oxidized to sulfate by sulfite oxidase, and then the sulfate is excreted into the urine by the kidney (Figure 1) [3, 22].
$\mathrm{Du}$ et al. first measured endogenous $\mathrm{SO}_{2}$ /AAT pathway in the cardiovascular system of Wistar rats and found that $\mathrm{SO}_{2}$ concentration in rat plasma was $15.54 \pm 1.68 \mu \mathrm{mol} / \mathrm{L}$ [14]. $\mathrm{Li}$ and Meng reported a similar sulfite level of $12.59 \pm$ $9.03 \mu \mathrm{mol} / \mathrm{L}$ in rat plasma [26]. The content of $\mathrm{SO}_{2}$ in aortic tissue was highest, up to $5.55 \pm 0.35 \mu \mathrm{mol} / \mathrm{g}$ protein, followed by pulmonary arteries $(3.27 \pm 0.21 \mu \mathrm{mol} / \mathrm{g}$ protein $)$, mesenteric arteries $(2.67 \pm 0.17 \mu \mathrm{mol} / g$ protein $)$, tail arteries $(2.50 \pm$ $0.20 \mu \mathrm{mol} / \mathrm{g}$ protein $)$, and renal arteries $(2.23 \pm 0.19 \mu \mathrm{mol} / \mathrm{g}$ protein), respectively [14]. Moreover, plasma AAT activity was $87 \pm 18 \mathrm{U} / \mathrm{L}$. Unlike $\mathrm{SO}_{2}$ content, the activity of AAT in the renal arteries was higher than that in other vascular tissues mentioned above [14]. Furthermore, AAT mRNA expression was rich in endothelial cells and in vascular smooth muscle cell (VSMC) beneath the endothelial layer [14].

\section{Physiological Effects of $\mathrm{SO}_{2}$ on the Cardiovascular System}

3.1. Vasorelaxant Effect of $\mathrm{SO}_{2} . \mathrm{SO}_{2}$ derivatives (mixture of sodium bisulfite and sodium sulfite, $1: 3 \mathrm{M} / \mathrm{M}$ in neutral 
solution) could induce a concentration-dependent relaxation of isolated rat aortic rings, whereas L-aspartate- $\beta$-hydroxamate (HDX), an inhibitor of $\mathrm{SO}_{2}$ synthase AAT, caused greater vasoconstriction than that of the control group [14]. And the vasorelaxing effects of $\mathrm{SO}_{2}$ gas and $\mathrm{SO}_{2}$ gas solution were similar $[27,28]$. Therefore, $\mathrm{SO}_{2}$ might act as a vasoactive molecule. It had a vital vasodilating function required for maintaining normal vascular tone.

The mechanisms of this physiological vasorelaxation by $\mathrm{SO}_{2}$ were complex. Nicardipine eliminated the vasorelaxing effect induced by $\mathrm{SO}_{2}$ derivatives, indicating that the L-type calcium $\left(\mathrm{L}-\mathrm{Ca}^{2+}\right.$ ) channel participated in the role of $\mathrm{SO}_{2}$ [14]. Additionally, at low concentration $(<450 \mu \mathrm{mol} / \mathrm{L})$, the vasorelaxing effect of $\mathrm{SO}_{2}$ was related to the big-conductance $\mathrm{Ca}^{2+}$-activated $\mathrm{K}^{+}\left(\mathrm{BK}_{\mathrm{Ca}}\right)$ channel, while at a high concentration $(>500 \mu \mathrm{mol} / \mathrm{L})$ the vasorelaxation induced by $\mathrm{SO}_{2}$ was associated to adenosine triphosphate-sensitive potassium $\left(\mathrm{K}_{\mathrm{ATP}}\right)$ channel activation and the $\mathrm{L}^{-\mathrm{Ca}^{2+}}$ channel [29]. Mechanistically, $\mathrm{SO}_{2}$ and its derivatives induced the $\mathrm{K}_{\text {ATP }}$ and $\mathrm{BK}_{\mathrm{Ca}}$ channels activation through increasing the expressions of Kir6.1, Kir6.2, SUR2B, and BK $\mathrm{Ca}_{\mathrm{C}}$ channel subunits $\alpha$ and $\beta 1$ in rat aortic rings, while $\mathrm{SO}_{2}$ and its derivatives inhibited the L-type calcium channel through decreasing the expressions of Cav1.2 and Cav1.3 [30]. Besides, $\mathrm{SO}_{2}$ derivatives increased levels of $3^{\prime}-5^{\prime}$-cyclic adenosine monophosphate (cAMP), prostacyclin $\left(\mathrm{PGI}_{2}\right)$, adenylyl cyclase $(\mathrm{AC})$ activity, and protein kinase $\mathrm{A}$ (PKA) activity in rat aortic rings, indicating that the relaxing effect of $\mathrm{SO}_{2}$ was related to the $\mathrm{PGI}_{2}-\mathrm{AC}$-cAMPPKA signal pathway [31, 32]. Moreover, the endothelial nitric oxide synthase- (eNOS-) nitric oxide- (NO-) $3^{\prime}-5^{\prime}$ cyclic guanosine monophosphate (cGMP) pathway and $\mathrm{BK}_{\mathrm{Ca}}$ channel partially mediated the vasorelaxing effect of $\mathrm{SO}_{2}$ and sodium bisulfite in an endothelium-dependent manner at low concentration $(<450 \mu \mathrm{M})$, while at high concentration $(\geq 1000 \mu \mathrm{M})$ the vasorelaxation induced by $\mathrm{SO}_{2}$ was endothelium independent and relied on the $\mathrm{K}_{\mathrm{ATP}}$ and $\mathrm{L}_{-} \mathrm{Ca}^{2+}$ channels $[26,33,34]$. Hence, ion channels, such as $\mathrm{L}^{-\mathrm{Ca}^{2+}}, \mathrm{K}_{\mathrm{ATP}}$, and $\mathrm{BK}_{\mathrm{Ca}}$ channels, as well as cGMP and cAMP pathways play important roles in the effects of $\mathrm{SO}_{2}$ on vasodilation.

3.2. Negative Inotropic Effect of $\mathrm{SO}_{2}$. In isolated perfused rat heart, gaseous $\mathrm{SO}_{2}$ and its derivatives $\left(\mathrm{NaHSO}_{3} / \mathrm{Na}_{2} \mathrm{SO}_{3}\right.$, 1:3 M/M, 0-2000 $\mu \mathrm{mol} / \mathrm{L}$ ) elicited a dose-dependent negative inotropic effect, which affected the heart rate, left ventricular developed pressure (LVDP), and the first derivatives of $\operatorname{LVDP}\left( \pm \mathrm{LV} d p / d t_{\max }\right)[35,36]$. And the gaseous $\mathrm{SO}_{2}$ induced a server negative effect compared to $\mathrm{SO}_{2}$ derivatives. The mechanisms for this inotropic effect are different between high concentration and low concentrations of $\mathrm{SO}_{2}$. At low concentrations, $\mathrm{SO}_{2}$ produced negative inotropic effects through upregulating the activities of protein kinase $\mathrm{C}(\mathrm{PKC})$, cyclooxygenase, and cGMP, while, at high concentrations, the inotropic effects induced by $\mathrm{SO}_{2}$ were associated with the activation of $\mathrm{K}_{\mathrm{ATP}}$ channel by increasing the expressions of Kir6.2 and SUR2A and the inhibition of calcium influx via the L-type calcium channel by decreasing the expressions of Cav1.2 and Cav1.3 in rat hearts [36, 37]. Moreover, $\mathrm{SO}_{2}$ could depress L-type calcium channel current in isolated rat cardiomyocytes [38]. These data indicated that $\mathrm{SO}_{2}$ had a negative inotropic effect on myocardial contractility and hemodynamic parameters, which might help to explain some cardiovascular effects induced by $\mathrm{SO}_{2}$.

\section{Pathophysiological Effects of $\mathrm{SO}_{2}$ on the Cardiovascular System}

4.1. $\mathrm{SO}_{2}$ and Hypertension. Hypertension is a major risk factor for many cardiovascular disorders. However, the pathogenesis of hypertension has not been fully elucidated. Exposure to $\mathrm{SO}_{2}$ (50 ppm, $6 \mathrm{hr} / \mathrm{d}, 5 \mathrm{~d} /$ wk for 31 weeks) was reported to cause a slight but consistent decrease in blood pressure in susceptible to salt-induced hypertension rats [39], implying that $\mathrm{SO}_{2}$ might regulate blood pressure. Moreover, spontaneously hypertensive rats (SHRs) exhibited a significant decrease in the plasma $\mathrm{SO}_{2}$ content and AAT activity in both serum and aorta [15]. And $\mathrm{SO}_{2}$ derivatives administration markedly inhibited the upregulated tail artery pressure of SHRs $[15,40]$, which suggested that $\mathrm{SO}_{2}$ played a role in the progress of hypertension. Arterial remodeling predominates in severe hypertension [41]. As well, $\mathrm{SO}_{2}$ alleviated the pressure to media, decreased the ratio of media to lumen radius, and reduced the proliferative index of smooth muscle cells in the thoracic aorta of SHRs compared to those of sterile water-treated rats [15]. These findings further verified that the inhibited endogenous $\mathrm{SO}_{2}$ /AAT pathway might participate in the development of hypertension. Vasorelaxation dysfunction is the main component of the pathogenesis of hypertension. $\mathrm{SO}_{2}$ could increase vasorelaxation in SHR arteries by enhancing the vasodilating response to $\mathrm{NO}$ in isolated aortic rings and promoting $\mathrm{NO}$ production of aortic tissues [40]. The interaction between $\mathrm{SO}_{2}$ and $\mathrm{NO}$ is involved in the mechanisms by which $\mathrm{SO}_{2}$ regulates hypertension.

The abnormally increased proliferation of VSMCs induces vascular remodeling and accelerates the development of hypertension [42]. Both exogenous $\mathrm{SO}_{2}$ derivatives and endogenous-derived $\mathrm{SO}_{2}$ by AAT overexpression significantly inhibited serum-stimulated VSMC proliferation through preventing cell cycle progression from G1 to $S$ phase and inhibiting DNA synthesis [43]. Further study demonstrated that $\mathrm{SO}_{2}$ elevated cellular cyclic adenosine monophosphate (cAMP) production to activate the PKA signaling, subsequently phosphorylated c-Raf on Ser259 site to block its activation, and then inhibited the extracellular regulated protein kinase (Erk)/mitogen-activated protein kinase (MAPK) signaling transduction, which finally prevented cell cycle progression and led to the suppression of VSMC proliferation [43]. The inhibition of VSMC proliferation might also be involved in $\mathrm{SO}_{2}$-mediated antihypertensive mechanisms.

\section{2. $\mathrm{SO}_{2}$ and Pulmonary Hypertension}

4.2.1. $\mathrm{SO}_{2}$ and Hypoxic Pulmonary Hypertension. Pulmonary hypertension, characterized by high pressure in pulmonary artery, is a common complication of congenital heart disease (CHD), ultimately inducing right ventricular failure and even death. A prospective cohort study showed that the serum 
$\mathrm{SO}_{2}$ levels of children were, respectively, $(10.6 \pm 2.4),(8.9 \pm$ $2.3),(7.3 \pm 2.9)$, and $(4.3 \pm 2.1) \mu \mathrm{M}$, in the control group, CHD without pulmonary hypertension group, CHD with mild pulmonary hypertension group, and CHD with moderate or severe pulmonary hypertension group [44], suggesting that a negative correlation existed between $\mathrm{SO}_{2}$ and pulmonary hypertension. Consistent with this, a downregulated $\mathrm{SO}_{2}$ level and AAT expression in lung tissue, accompanied with significant pulmonary hypertension, pulmonary vascular remodeling, and increased vascular inflammation, were found in rats under hypoxic condition [16, 45]. Most importantly, $\mathrm{SO}_{2}$ derivatives could markedly lower mean pulmonary artery pressure (mPAP) of hypoxic pulmonary hypertensive rats, whereas HDX advanced pulmonary hypertension $[16,45]$, indicating that decreased $\mathrm{SO}_{2} / \mathrm{AAT}$ pathway was involved in the development on hypoxic pulmonary hypertension. The hallmark pathological feature of hypoxic pulmonary hypertension is the pulmonary vascular structural remodeling including extracellular matrix accumulation, vascular smooth muscle proliferation, and inflammatory cells infiltrates [46]. $\mathrm{SO}_{2}$ derivatives prevented pulmonary vascular remodeling in hypoxic pulmonary hypertension through promoting collagen I and III degradation, suppressing abnormal collagen deposition in pulmonary vascular walls and through inhibiting pulmonary arterial SMC proliferation by downregulating Raf-1, MEK-1, and phosphorylating ERK under hypoxia [16]. Inflammation is important in the pathogenesis of hypoxic pulmonary hypertension. In addition, $\mathrm{SO}_{2}$ could inhibit pulmonary inflammation by suppressing expressions of nuclear factor-kappa $\mathrm{B}$ (NF- $\kappa \mathrm{B}$ ) and intercellular adhesion molecule-1 (ICAM-1) [16], indicating the inhibitory effects of $\mathrm{SO}_{2}$ on inflammation may also be involved in the mechanism by which $\mathrm{SO}_{2}$ protects against hypoxic pulmonary hypertension.

4.2.2. $\mathrm{SO}_{2}$ and Monocrotaline-Induced Pulmonary Hypertension. Monocrotaline (MCT), a pyrrolizidine alkaloid, increased $\mathrm{mPAP}$ and the ratio of right ventricle to left ventricle plus septum, coincident with the elevated $\mathrm{SO}_{2}$ content, AAT activity, and expression in rats [47]. $\mathrm{SO}_{2}$ derivatives injection significantly lowered mPAP and alleviated small and median pulmonary artery structural remodeling, whereas HDX which inhibited the activity of AAT and the production of endogenous $\mathrm{SO}_{2}$ further augmented mPAP, promoted right ventricular hypertrophy, and worsened pulmonary arteries structural remodeling [47]. These findings implied that the upregulation of endogenous $\mathrm{SO}_{2}$ /AAT pathway might play a protective role in the development of MCT-induced pulmonary hypertension. The enhancement of oxidative stress is one of the main pathogenesis of MCT-induced pulmonary hypertension [48]. $\mathrm{SO}_{2}$ could upregulate the activities of antioxidative enzymes, including superoxide dismutase (SOD), glutathione peroxidase (GSH-Px), and catalase (CAT) in lung tissues and plasma from MCT-induced pulmonary hypertensive rats, whereas HDX decreased the activities of antioxidative enzymes [47]. These data suggested that the promotion of endogenous antioxidative capacities might be responsible for the protective role of $\mathrm{SO}_{2}$ in MCTinduced pulmonary hypertension.
4.2.3. $\mathrm{SO}_{2}$ and High Pulmonary Blood Flow-Induced Pulmonary Hypertension. Severe pulmonary hypertension develops secondary to high pulmonary blood flow in patients with left-to-right shunt congenital heart defects or systemic arteriovenous shunt $[49,50]$. However, the underlying mechanisms for flow-induced pulmonary hypertension remain poorly understood. The endogenous $\mathrm{SO}_{2}$ /AAT2 pathway in pulmonary tissues was also inhibited in rats with pulmonary hypertension induced by high pulmonary blood flow [51]. $\mathrm{SO}_{2}$ reduced systolic pulmonary arterial pressure and improved pulmonary arterial structural remodeling, exhibiting decreased ratio of muscularized arteries to small pulmonary arteries and increased percentage of nonmuscularized arteries in the development of high pulmonary blood flowinduced pulmonary hypertension [51]. The mechanism was unclear, however. Both $\mathrm{SO}_{2}$ and $\mathrm{H}_{2} \mathrm{~S}$ were derived from the methionine metabolism and they could convert to each other in mammals. Moreover, the endogenous $\mathrm{H}_{2} \mathrm{~S}$ pathway exerted obvious mitigation effect on pulmonary hypertension induced by high pulmonary blood flow and $\mathrm{H}_{2} \mathrm{~S}$ had strong vasodilating effect. Therefore, the researchers investigated the impact of $\mathrm{SO}_{2}$ on the endogenous $\mathrm{H}_{2} \mathrm{~S}$ generating pathway in the pathogenesis of high blood flow-induced pulmonary hypertension. And they found that $\mathrm{SO}_{2}$ derivatives could upregulate the concentration of $\mathrm{H}_{2} \mathrm{~S}$ in lung tissues, as well as the expressions of the key generating enzymes of $\mathrm{H}_{2} \mathrm{~S}$, including cystathionine $\gamma$-lyase (CSE), cystathionine $\beta$ synthase (CBS), and 3-mercaptopyruvate sulfurtransferase (3MST) [51]. Furthermore, $\mathrm{SO}_{2}$ increased the protein expression of these $\mathrm{H}_{2} \mathrm{~S}$ producing enzymes probably through upregulating their gene transcription. These data suggested that $\mathrm{SO}_{2}$ alleviated pulmonary hypertension induced by high pulmonary blood flow in association with upregulating the reduced endogenous $\mathrm{H}_{2} \mathrm{~S}$ pathway.

4.3. $\mathrm{SO}_{2}$ and Atherosclerosis. Atherosclerosis, a chronic and progressive pathological process in arteries, is a crucial pathological manifestation of cardiovascular diseases. Vascular inflammation, oxidative stress, VSMC proliferation, endothelial injury, and foam cell accumulation contribute to the formation of atherosclerotic plaque. Environmental toxicological study showed that the chronic exposure to gaseous air pollution such as $\mathrm{SO}_{2}, \mathrm{NO}$, and $\mathrm{CO}$ might lead to the promotion of atherosclerosis [52, 53]. Growing evidence demonstrated that endogenous $\mathrm{NO}, \mathrm{CO}$, and $\mathrm{H}_{2} \mathrm{~S}$ were beneficial in alleviating atherosclerosis [54-56]. They exerted significant anti-inflammation effect in the development of atherosclerosis, especially endothelium-derived NO which played a notably protective role in the early stage of atherosclerosis. However, the role of $\mathrm{SO}_{2}$ at physiological concentration in the development of atherosclerosis was unclear. $\mathrm{Li}$ et al. found that plasma and aortic $\mathrm{SO}_{2}$ contents were downregulated with the reduced aortic AAT activity in atherosclerosis rats [17], implying that the inhibition of $\mathrm{SO}_{2} / \mathrm{AAT}$ pathway might be involved in the pathogenesis of atherosclerosis. $\mathrm{SO}_{2}$ derivatives treatment diminished the size of atherosclerotic plaques in the coronary artery, not only by increasing $\mathrm{H}_{2} \mathrm{~S} / \mathrm{CSE}$ pathway and the $\mathrm{NO}$ /nitric oxide 
synthase (NOS) pathway, but also by elevating the antioxidative capacities through increasing plasma GSH-Px and SOD activities and decreasing MDA level [17]. Additionally, suppression of VSMC proliferation via cAMP/PKA signalingmediated Erk/MAPK pathway might also contribute to the antiatherosclerotic effects of $\mathrm{SO}_{2}$ [43].

4.4. $\mathrm{SO}_{2}$ and Myocardial Ischemia Reperfusion. Myocardial ischemia-reperfusion (I/R) injury is an important cause of tissue and cell injury and often leads to heart failure. The main mechanisms involve inflammation, oxidative damage, and intracellular and mitochondrial calcium overload [57]. In rat myocardial I/R models made by ligating the left coronary artery for $30 \mathrm{~min}$ and reperfusion for $120 \mathrm{~min}$, AAT1 protein expression was significantly decreased compared to sham operation group [18]. And $\mathrm{SO}_{2}$ derivatives preconditioning for $10 \mathrm{~min}$ before ischemia (with a low concentration of sulfur dioxide of $1-10 \mu \mathrm{mol} / \mathrm{kg}$ ) significantly decreased myocardial infract size and lowered levels of myocardial enzymes creatine kinase (CK) and lactate dehydrogenase (LDH) in plasma of rats with $\mathrm{I} / \mathrm{R}$ injury in vivo [18]. $\mathrm{SO}_{2}$ preconditioning also increased cardiac function and attenuated myocardium apoptosis induced by $\mathrm{I} / \mathrm{R}$ [18]. Ischemic preconditioninginduced endoplasmic reticulum stress (ERS) plays a protective role in the ischemia injury. Glucose-regulated protein 78 (GRP78), C/EBP homologous protein (CHOP), and phosphorylated eukaryotic initiation of the factor $2 \alpha$-subunit (peIF $2 \alpha$ ) are the markers of myocardial ERS. $\mathrm{SO}_{2}$ pretreatment induced myocardial GRP78 expression and eIF2 $\alpha$ phosphorylation prior to myocardial $\mathrm{I} / \mathrm{R}$, while inhibiting expressions of myocardial GRP78, CHOP, and p-eIF2 $\alpha$ in rats with myocardial I/R [18]. Dithiothreitol, an ERS activator [58], mimicked the cardioprotective effect of $\mathrm{SO}_{2}$, whereas ERS inhibitor 4-phenylbutyrate abolished the cardioprotection of $\mathrm{SO}_{2}$ preconditioning $[18,59]$. The above data suggested that augmentation of ERS by $\mathrm{SO}_{2}$ preconditioning before myocardial I/R contributed to cardioprotection against lethal ischemia. Moreover, $\mathrm{SO}_{2}$ preconditioning significantly elevated the phosphorylation of Akt and PI3K p85 and attenuated the myocardial damage in rats with I/R injury [60]. LY294002, a PI3K inhibitor, prevented the protective function of $\mathrm{SO}_{2}$ preconditioning as well as $\mathrm{SO}_{2}$-induced GRP78 and p-eIF2 $\alpha$ expression $[18,60]$, indicating that PI3K/Akt signaling pathway likely mediated the activation of ERS by $\mathrm{SO}_{2}$ pretreatment in rats subjected to myocardial I/R. In addition, oxidative stress is involved in the pathogenesis of myocardial I/R. $\mathrm{SO}_{2}$ preconditioning with low dose of $\mathrm{SO}_{2}(1$ and $5 \mu \mathrm{mol} / \mathrm{kg})$ prior to ischemia could significantly elevate plasma levels of SOD, GSH, and GSH-Px and reduce the MDA level [61], indicating that $\mathrm{SO}_{2}$ preconditioning enhanced the antioxidative capacity in rats with myocardial I/R. MAPK signaling, one of the most important pathways in cell signal transduction, is crucial to myocardial I/R. $\mathrm{SO}_{2}$ preconditioning significantly improved cardiac function and reduced myocardial expression of phosphorylated ERK1/2 protein in isolated rat heart with I/R [62]. Pretreated with PD98059, the ERK1/2 inhibitor abolished the above functions of $\mathrm{SO}_{2}$ [62]. These data indicated that inhibition of ERK1/2 signal pathway activation mediated the cardioprotection of $\mathrm{SO}_{2}$ preconditioning in isolated rat heart subjected to I/R. Taken together, elevation of PI3K/AKT signaling, suppression of ERK-MAPK pathway, augmentation of ERS, enhancement of antioxidative capacity, and attenuation of cardiomyocyte apoptosis might be involved in $\mathrm{SO}_{2}$-mediated cardiac protective mechanisms.

4.5. $\mathrm{SO}_{2}$ and Myocardial Injury. Myocardial injury is a common feature in various cardiac diseases. The underlying mechanisms include hypoxia, overactive oxidative stress, and calcium overload. A previous study found that endogenous $\mathrm{SO}_{2}$ /AAT pathway was downregulated in isoproterenol(ISO-) induced myocardial injury in rats [63]. Administration of $\mathrm{SO}_{2}(85 \mathrm{mg} /(\mathrm{kg}$ day $))$ could alleviate cardiac dysfunction and myocardial damage induced by ISO [63]. These data demonstrated that endogenous $\mathrm{SO}_{2}$ might be an important regulator in the pathophysiological process of myocardial injury. The molecular mechanisms underlying the cardioprotective effects of $\mathrm{SO}_{2}$ were still unknown. Oxidative stress was involved in the pathogenesis for ISO-induced myocardial injury. ISO produced oxygen free radicals caused membrane lipid peroxidation, injured the structure of cardiomyocytes, and finally resulted in myocardial damage [64]. But $\mathrm{SO}_{2}$ could increase myocardial antioxidant capacity in rats with myocardial injury by increasing the myocardial activity of SOD and GSH, upregulating the mRNA expression of SOD2 and GSH-Pxl, and decreasing products of oxidative stress such as $\mathrm{H}_{2} \mathrm{O}_{2}$ and $\mathrm{O}_{2}{ }^{--}$[63]. Oxidative stress could cause ERS in rat cardiomyocytes [65]. And the overactivated ERS would contribute to the development of myocardial injury. $\mathrm{SO}_{2}$ significantly inhibited the excessive activation of ERS, which might be involved in the mechanism by which $\mathrm{SO}_{2}$ derivatives protected against myocardial injury induced by ISO [66]. In addition, the products of oxidative stress cause the cardiomyocyte membrane damage and morphological mitochondrial injury. $\mathrm{SO}_{2}$ also attenuated ISO-induced mitochondrial swelling and deformation, which was important feature in apoptosis [63]. And cardiomyocyte apoptosis is a key pathological change in myocardial injury. Of note, supplementation of $\mathrm{SO}_{2}$ derivatives alleviated ISO-induced myocardial injury partly through reducing cardiomyocyte apoptosis [67]. The antiapoptotic function of $\mathrm{SO}_{2}$ was mediated by promoting bcl-2 expression, suppressing bax expression, enhancing mitochondrial membrane potential, inhibiting mitochondrion MPTP opening, reducing the release of cytochrome $\mathrm{c}$ from mitochondrion into cytoplasm, and decreasing the activation of caspase- 9 and caspase-3 [67]. Therefore, the bcl-2/cytc/caspase- $9 /$ caspase- 3 pathway was involved in the ISO-induced myocardial injury in rats. Intracellular calcium homeostasis exerts a fundamental effect on myocardial physiology and pathology. And calcium overload is an important mechanism involved in myocardial injury. $\mathrm{SO}_{2}$ treatment could inhibit the increased intracellular free $\mathrm{Ca}^{2+}$ concentration induced by ISO in H9C2 cells [68], indicating that the protective effect of $\mathrm{SO}_{2}$ in myocardial injury might be related to the calcium homeostasis regulated by $\mathrm{SO}_{2}$ in cardiomyocytes. Moreover, $\mathrm{SO}_{2}$ derivatives 
could modulate L-type calcium current and voltage-gated potassium channels in rat cardiomyocytes, indicating that ion channels might also be involved in the effect of $\mathrm{SO}_{2}$ on cardiomyocyte injury $[69,70]$.

\section{Interaction among $\mathrm{SO}_{2}$ and Other Gasotransmitters}

$\mathrm{SO}_{2}$ and $\mathrm{H}_{2} \mathrm{~S}$ share the same endogenous substrate Lcysteine, and they can transform into each other under some biochemical condition [6, 23, 71]. Moreover, they share similar regulatory roles including vasorelaxation, antioxidative action, and inhibition of inflammation and apoptosis. Chen et al. found that $\mathrm{SO}_{2}$ upregulated the concentration and production of $\mathrm{H}_{2} \mathrm{~S}$ in hypoxic rats. And $\mathrm{SO}_{2}$ increased the expression of CSE and 3MST in pulmonary arteries of hypoxic pulmonary hypertensive rats [72]. In addition, $\mathrm{SO}_{2}$ alleviated pulmonary hypertension and improved the pulmonary vascular pathological injury induced by high pulmonary blood flow in association with upregulating the endogenous $\mathrm{H}_{2} \mathrm{~S}$ pathway [51]. Furthermore, $\mathrm{SO}_{2}$ derivatives have a marked antiatherogenic effect with an increased aortic $\mathrm{H}_{2} \mathrm{~S} / \mathrm{CSE}$ pathway in atherosclerotic rats [17]. In rats with myocardial I/R injury, $\mathrm{SO}_{2}$ preconditioning markedly upregulated the myocardial $\mathrm{H}_{2} \mathrm{~S}$ level and CSE expression [61]. The above findings provide some evidence that there is a crosstalk between $\mathrm{SO}_{2}$ and $\mathrm{H}_{2} \mathrm{~S}$. Moreover, $\mathrm{NO}$ also shares a variety of the similar biological effects of $\mathrm{H}_{2} \mathrm{~S}$ and $\mathrm{SO}_{2}$, including vasodilation, antioxidation, and anti-inflammatory actions. $\mathrm{Li}$ and Meng found that a low concentration ( 3 or $5 \mathrm{nM}$ ) of a NO donor sodium-nitroprusside enhanced the vasodilating effect of $\mathrm{SO}_{2}$ by nearly sixfold [26], suggesting that $\mathrm{SO}_{2}$ and $\mathrm{NO}$ have a synergistic effect on vasodilation. In contrast, the NOS inhibitor L-NAME could abolish the vasorelaxing effect of $\mathrm{SO}_{2}$ derivatives $(0.5$ and $1 \mathrm{mM})$ in endothelium-intact rings, indicating that endothelium-dependent vasorelaxation induced by $\mathrm{SO}_{2}$ was partially mediated by a NOS pathway [73]. Additionally, both acute and prolonged $\mathrm{SO}_{2}$ exposure upregulated the eNOS-NO-cGMP pathway, which might be involved in the vasodilation induced by $\mathrm{SO}_{2}$ [34]. Moreover, $\mathrm{SO}_{2}$ increased vasorelaxation in SHRs by enhancing the vasorelaxing response to $\mathrm{NO}$ and upregulating $\mathrm{NO}$ production in aortic tissues [40]. And $\mathrm{SO}_{2}$ also increased NO/NOS pathway in rats with atherosclerosis [17]. By contrast, $\mathrm{SO}_{2}$ pretreatment reduced the myocardial tissue levels of $\mathrm{NO}$ and expression of iNOS in rats with I/R [61]. These data suggest that there is also an interaction between $\mathrm{SO}_{2}$ and NO. Hence, endogenous $\mathrm{SO}_{2}$ participates in crosstalk with $\mathrm{H}_{2} \mathrm{~S}$ and $\mathrm{NO}$ and an endogenous gaseous messenger molecule network might exist in mammals. However, there are still many questions to be answered about the interactions among these gases. For example, the exact pertinence among these gases in the various pathways of cardiovascular protection has not been fully explored. It is also not known if a combination of these gases will provide synergistic effects in the therapy of cardiovascular diseases. Therefore, additional studies are needed to further investigate interactions among the gasotransmitter pathways.

\section{Conclusions}

In summary, $\mathrm{SO}_{2}$ can be generated in the cardiovascular system of mammals and the $\mathrm{SO}_{2}$ /AAT pathway participates in many biological functions [22, 74]. Endogenously derived $\mathrm{SO}_{2}$ or $\mathrm{SO}_{2}$ derivatives at physiological concentrations play a crucial role in normal physiological process including regulation of vascular tone and cardiac function. In addition, $\mathrm{SO}_{2}$ /AAT pathway has important pathophysiological significance in many cardiovascular diseases, such as hypertension, pulmonary hypertension, atherosclerosis, ischemiareperfusion injury, and myocardial injury. Just as NO, carbon monoxide $(\mathrm{CO})$, and $\mathrm{H}_{2} \mathrm{~S}, \mathrm{SO}_{2}$ is also an endogenous gaseous signaling molecule in the cardiovascular system [71, 75]. However, the biological mechanisms by which endogenous $\mathrm{SO}_{2}$ regulates different cardiovascular diseases and the further cardiovascular effects of $\mathrm{SO}_{2}$ still need to be deeply investigated.

Clarifying the interactions among $\mathrm{SO}_{2}$ and other endogenous gasotransmitters could improve clinical translation. $\mathrm{SO}_{2}$ could upregulate endogenous level of $\mathrm{H}_{2} \mathrm{~S}$ or $\mathrm{NO}$ in several cardiovascular diseases such as atherosclerosis, systemic hypertension, or pulmonary hypertension [17, 40, 51]. These lines of evidence imply a crosstalk among $\mathrm{SO}_{2}$ and other gasotransmitters ( $\mathrm{NO}, \mathrm{CO}$, and $\mathrm{H}_{2} \mathrm{~S}$ ) in the cardiovascular system, which requires further exploration.

An understanding of the cardiovascular protective function of $\mathrm{SO}_{2}$ may lead to a new therapeutic strategy based on the modulation of $\mathrm{SO}_{2}$ production. Thus, the function and signaling pathway relating to AAT in the cardiovascular system are worthy of further investigation. Additionally, the design of $\mathrm{SO}_{2}$-controlled releasing agents under physiological condition is extremely urgent, because the stable and reliable $\mathrm{SO}_{2}$ donors are not only the useful research tools, but also potential therapeutic agents to treat cardiovascular diseases. Nowadays, the majority of cardiovascular studies on $\mathrm{SO}_{2}$ have been performed in rats and mice, which lack clinical evidence. Exploring the role of $\mathrm{SO}_{2}$ in large animal models with similar cardiovascular features as human suffering from cardiovascular diseases would help a transition to clinical trials.
Abbreviations
$\mathrm{SO}_{2}: \quad$ Sulfur dioxide
AAT: Aspartate aminotransferase
I/R: Ischemia-reperfusion
CDO: Cysteine dioxygenase
$\mathrm{H}_{2} \mathrm{~S}$ : Hydrogen sulfide
NO: $\quad$ Nitric oxide
CO: $\quad$ Carbon monoxide
HDX: L-Aspartate- $\beta$-hydroxamate

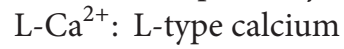
$\mathrm{BK}_{\mathrm{Ca}}$ : Big-conductance $\mathrm{Ca}^{2+}$-activated $\mathrm{K}^{+}$
$\mathrm{K}_{\mathrm{ATP}}$ : Adenosine triphosphate-sensitive potassium
cAMP: $3^{\prime}-5^{\prime}$-Cyclic adenosine monophosphate
$\mathrm{PGI}_{2}$ : Prostacyclin
AC: Adenylyl cyclase
PKA: Protein kinase A
cGMP: $3^{\prime}-5^{\prime}$-Cyclic guanosine monophosphate 
LVDP: $\quad$ Left ventricular developed pressure

PKC: $\quad$ Protein kinase C

SHR: $\quad$ Spontaneously hypertensive rats

eNOS: $\quad$ Nitric oxide synthase

VSMCs: Vascular smooth muscle cells

PDGF-BB: Platelet-derived growth factor-BB

Erk 1/2: $\quad$ Extracellular regulated protein kinase 1/2

MAPK: Mitogen-activated protein kinase

CHD: $\quad$ Congenital heart defects

mPAP: $\quad$ Mean pulmonary artery pressure

NF- $\kappa$ B: $\quad$ Nuclear factor-kappa B

ICAM-1: Intercellular adhesion molecule-1

MCT: $\quad$ Monocrotaline

SOD: $\quad$ Superoxide dismutase

GSH-Px: Glutathione peroxidase

CAT: Catalase

CSE: $\quad$ Cystathionine $\gamma$-lyase

CBS: $\quad$ Cystathionine $\beta$-synthase

3MST: $\quad 3$-Mercaptopyruvate sulfurtransferase

NOS: $\quad$ Nitric oxide synthase

CK: $\quad$ Creatine kinase

LDH: $\quad$ Lactate dehydrogenase

GRP78: $\quad$ Glucose-regulated protein 78

CHOP: $\quad$ C/EBP homologous protein

eIF2 $\alpha$ : Eukaryotic initiation of the factor $2 \alpha$ subunit

p-eIF2 $\alpha$ : Phosphorylated eIF2 $\alpha$

ERS: $\quad$ Endoplasmic reticulum stress

ISO: Isoproterenol.

\section{Conflict of Interests}

The authors declare that there is no conflict of interests regarding the publication of this paper.

\section{Acknowledgment}

This work was supported by the National Natural Science Foundation of China (Grants nos. 81400311, 31440052, and 91439110).

\section{References}

[1] A. L. Woerman and D. Mendelowitz, "Perinatal sulfur dioxide exposure alters brainstem parasympathetic control of heart rate," Cardiovascular Research, vol. 99, no. 1, pp. 16-23, 2013.

[2] K.-B. Min, J.-Y. Min, S.-I. Cho, and D. Paek, “The relationship between air pollutants and heart-rate variability among community residents in Korea," Inhalation Toxicology, vol. 20, no. 4, pp. 435-444, 2008.

[3] T. P. Singer and E. B. Kearney, "Intermediary metabolism of Lcysteinesulfinic acid in animal tissues," Archives of Biochemistry and Biophysics, vol. 61, no. 2, pp. 397-409, 1956.

[4] L. Li and P. K. Moore, "An overview of the biological significance of endogenous gases: new roles for old molecules," Biochemical Society Transactions, vol. 35, no. 5, pp. 1138-1141, 2007.

[5] D. E. Barañano, C. D. Ferris, and S. H. Snyder, "Atypical neural messengers," Trends in Neurosciences, vol. 24, no. 2, pp. 99-106, 2001.
[6] R. Shapiro, "Genetic effects of bisulfite (sulfur dioxide)," Mutation Research, vol. 39, no. 2, pp. 149-175, 1977.

[7] C. Mottley, T. B. Trice, and R. P. Mason, "Direct detection of the sulfur trioxide radical anion during the horseradish peroxidase-hydrogen peroxide oxidation of sulfite (aqueous sulfur dioxide)," Molecular Pharmacology, vol. 22, no. 3, pp. 732737, 1982.

[8] G. A. Reed, M. J. Ryan, and K. S. Adams, "Sulfite enhancement of diolepoxide mutagenicity: the role of altered glutathione metabolism," Carcinogenesis, vol. 11, no. 9, pp. 1635-1639, 1990.

[9] A. J. Ji, S. R. Savon, and D. W. Jacobsen, "Determination of total serum sulfite by HPLC with fluorescence detection," Clinical Chemistry, vol. 41, no. 6, part 1, pp. 897-903, 1995.

[10] H. Kajiyama, Y. Nojima, H. Mitsuhashi et al., "Elevated levels of serum sulfite in patients with chronic renal failure," Journal of the American Society of Nephrology, vol. 11, no. 5, pp. 923-927, 2000.

[11] H. Mitsuhashi, H. Ikeuchi, S. Yamashita et al., "Increased levels of serum sulfite in patients with acute pneumonia," Shock, vol. 21, no. 2, pp. 99-102, 2004.

[12] W. S. Wu, Y. R. Jia, S. X. Du, H. Tang, Y. L. Sun, and L. M. Sun, "Changes of sulfur dioxide, nuclear factor- $\kappa \mathrm{B}$, and interleukin- 8 levels in pediatric acute lymphoblastic leukemia with bacterial inflammation," Chinese Medical Journal, vol. 127, no. 23, pp. 4110-4113, 2014.

[13] M. Balazy, I. A. Abu-Yousef, D. N. Harpp, and J. Park, "Identification of carbonyl sulfide and sulfur dioxide in porcine coronary artery by gas chromatography/mass spectrometry, possible relevance to EDHF," Biochemical and Biophysical Research Communications, vol. 311, no. 3, pp. 728-734, 2003.

[14] S.-X. Du, H.-F. Jin, D.-F. Bu et al., "Endogenously generated sulfur dioxide and its vasorelaxant effect in rats," Acta Pharmacologica Sinica, vol. 29, no. 8, pp. 923-930, 2008.

[15] X. Zhao, H.-F. Jin, C.-S. Tang, and J.-B. Du, "Effects of sulfur dioxide, on the proliferation and apoptosis of aorta smooth muscle cells in hypertension: experiments with rats," Zhonghua Yi Xue Za Zhi, vol. 88, no. 18, pp. 1279-1283, 2008.

[16] Y. Sun, Y. Tian, M. Prabha et al., "Effects of sulfur dioxide on hypoxic pulmonary vascular structural remodeling," Laboratory Investigation, vol. 90, no. 1, pp. 68-82, 2010.

[17] W. Li, C. Tang, H. Jin, and J. Du, "Regulatory effects of sulfur dioxide on the development of atherosclerotic lesions and vascular hydrogen sulfide in atherosclerotic rats," Atherosclerosis, vol. 215, no. 2, pp. 323-330, 2011.

[18] X.-B. Wang, X.-M. Huang, T. Ochs et al., "Effect of sulfur dioxide preconditioning on rat myocardial ischemia/reperfusion injury by inducing endoplasmic reticulum stress," Basic Research in Cardiology, vol. 106, no. 5, pp. 865-878, 2011.

[19] X. Li, F. W. Bazer, H. Gao et al., "Amino acids and gaseous signaling," Amino Acids, vol. 37, no. 1, pp. 65-78, 2009.

[20] Z.-Q. Meng and J.-L. Li, "Progress in sulfur dioxide biology: from toxicology to physiology," Sheng Li Xue Bao, vol. 63, no. 6, pp. 593-600, 2011.

[21] H. Tian, "Advances in the study on endogenous sulfur dioxide in the cardiovascular system," Chinese Medical Journal, vol. 127, no. 21, pp. 3803-3807, 2014.

[22] M. H. Stipanuk, "Metabolism of sulfur-containing amino acids," Annual Review of Nutrition, vol. 6, pp. 179-209, 1986.

[23] H. Mitsuhashi, S. Yamashita, H. Ikeuchi et al., "Oxidative stressdependent conversion of hydrogen sulfide to sulfite by activated neutrophils," Shock, vol. 24, no. 6, pp. 529-534, 2005. 
[24] P. Kamoun, "Endogenous production of hydrogen sulfide in mammals," Amino Acids, vol. 26, no. 3, pp. 243-254, 2004.

[25] K. Qu, S. W. Lee, J. S. Bian, C.-M. Low, and P. T.-H. Wong, "Hydrogen sulfide: neurochemistry and neurobiology," Neurochemistry International, vol. 52, no. 1, pp. 155-165, 2008.

[26] J. Li and Z. Meng, "The role of sulfur dioxide as an endogenous gaseous vasoactive factor in synergy with nitric oxide," Nitric Oxide, vol. 20, no. 3, pp. 166-174, 2009.

[27] Z. Meng, J. Li, Q. Zhang et al., "Vasodilator effect of gaseous sulfur dioxide and regulation of its level by Ach in rat vascular tissues," Inhalation Toxicology, vol. 21, no. 14, pp. 1223-1228, 2009.

[28] Z. Meng, H. Geng, J. Bai, and G. Yan, "Blood pressure of rats lowered by sulfur dioxide and its derivatives," Inhalation Toxicology, vol. 15, no. 9, pp. 951-959, 2003.

[29] Q. Zhang and Z. Meng, "The vasodilator mechanism of sulfur dioxide on isolated aortic rings of rats: involvement of the $\mathrm{K}^{+}$ and $\mathrm{Ca}^{2+}$ channels," European Journal of Pharmacology, vol. 602, no. 1, pp. 117-123, 2009.

[30] Q. Zhang, J. Tian, Y. Bai et al., "Effects of gaseous sulfur dioxide and its derivatives on the expression of $\mathrm{K}_{\mathrm{ATP}}, \mathrm{BK}_{\mathrm{Ca}}$ and ${\mathrm{L}-\mathrm{Ca}^{2+}}^{2+}$ channels in rat aortas in vitro," European Journal of Pharmacology, vol. 742, pp. 31-41, 2014.

[31] Z. Meng, Y. Li, and J. Li, "Vasodilatation of sulfur dioxide derivatives and signal transduction," Archives of Biochemistry and Biophysics, vol. 467, no. 2, pp. 291-296, 2007.

[32] Z. Meng and H. Zhang, "The vasodilator effect and its mechanism of sulfur dioxide-derivatives on isolated aortic rings of rats," Inhalation Toxicology, vol. 19, no. 11, pp. 979-986, 2007.

[33] Z. Meng, Z. Yang, J. Li, and Q. Zhang, “The vasorelaxant effect and its mechanisms of sodium bisulfite as a sulfur dioxide donor," Chemosphere, vol. 89, no. 5, pp. 579-584, 2012.

[34] J. Li, R. Li, and Z. Meng, "Sulfur dioxide upregulates the aortic nitric oxide pathway in rats," European Journal of Pharmacology, vol. 645, no. 1-3, pp. 143-150, 2010.

[35] S. Q. Zhang, J. B. Du, Y. Tian, B. Geng, C. S. Tang, and X. Y. Tang, "Effects of sulfur dioxide on cardiac function of isolated perfusion heart of rat," Zhonghua Yi Xue Za Zhi, vol. 88, no. 12, pp. 830-834, 2008.

[36] Q. Zhang and Z. Meng, "The negative inotropic effects of gaseous sulfur dioxide and its derivatives in the isolated perfused rat heart," Environmental Toxicology, vol. 27, no. 3, pp. 175184, 2012.

[37] Q. Zhang, Y. Bai, Z. Yang, J. Tian, and Z. Meng, "Effect of sulfur dioxide inhalation on the expression of $\mathrm{K}_{\mathrm{ATP}}$ and L$\mathrm{Ca}^{2+}$ channels in rat hearts," Environmental Toxicology and Pharmacology, vol. 39, no. 3, pp. 1132-1138, 2015.

[38] R.-Y. Zhang, J.-B. Du, Y. Sun et al., "Sulfur dioxide derivatives depress L-type calcium channel in rat cardiomyocytes," Clinical and Experimental Pharmacology and Physiology, vol. 38, no. 7, pp. 416-422, 2011.

[39] R. T. Drew, R. S. Kutzman, D. L. Costa, and J. Iwai, "Effects of sulfur dioxide and ozone on hypertension sensitive and resistant rats," Fundamental and Applied Toxicology, vol. 3, no. 4, pp. 298-302, 1983.

[40] W. Lu, Y. Sun, C. Tang et al., "Sulfur dioxide derivatives improve the vasorelaxation in the spontaneously hypertensive rat by enhancing the vasorelaxant response to nitric oxide," Experimental Biology and Medicine, vol. 237, no. 7, pp. 867-872, 2012.
[41] H. D. Intengan and E. L. Schiffrin, "Vascular remodeling in hypertension: roles of apoptosis, inflammation, and fibrosis," Hypertension, vol. 38, no. 3, pp. 581-587, 2001.

[42] Y. C. Fung, "What are the residual stresses doing in our blood vessels?" Annals of Biomedical Engineering, vol. 19, no. 3, pp. 237-249, 1991.

[43] D. Liu, Y. Huang, D. Bu et al., "Sulfur dioxide inhibits vascular smooth muscle cell proliferation via suppressing the Erk/MAP kinase pathway mediated by cAMP/PKA signaling," Cell Death \& Disease, vol. 5, Article ID e1251, 2014.

[44] R. Yang, Y. Yang, X. Dong, X. Wu, and Y. Wei, "Correlation between endogenous sulfur dioxide and homocysteine in children with pulmonary arterial hypertension associated with congenital heart disease," Zhonghua Er Ke Za Zhi, vol. 52, no. 8, pp. 625-629, 2014.

[45] Y. Tian, X.-Y. Tang, H.-F. Jin, C.-S. Tang, and J.-B. Du, "Effect of sulfur dioxide on pulmonary vascular structure of hypoxic pulmonary hypertensive rats," Chinese Journal of Pediatrics, vol. 46, no. 9, pp. 675-679, 2008.

[46] V. Amsellem, L. Lipskaia, S. Abid et al., "CCR5 as a treatment target in pulmonary arterial hypertension," Circulation, vol. 130, no. 11, pp. 880-891, 2014.

[47] H.-F. Jin, S.-X. Du, X. Zhao et al., "Effects of endogenous sulfur dioxide on monocrotaline-induced pulmonary hypertension in rats," Acta Pharmacologica Sinica, vol. 29, no. 10, pp. 1157-1166, 2008.

[48] S. M. Aziz, M. Toborek, B. Hennig, E. Endean, and D. W. Lipke, "Polyamine regulatory processes and oxidative stress in monocrotaline-treated pulmonary artery endothelial cells," Cell Biology International, vol. 21, no. 12, pp. 801-812, 1997.

[49] J. I. E. Hoffman, A. M. Rudolph, and M. A. Heymann, "Pulmonary vascular disease with congenital heart lesions: pathologic features and causes," Circulation, vol. 64, no. 5, pp. 873877, 1981.

[50] C.-F. Lam, T. E. Peterson, A. J. Croatt, K. A. Nath, and Z. S. Katusic, "Functional adaptation and remodeling of pulmonary artery in flow-induced pulmonary hypertension," The American Journal of Physiology-Heart and Circulatory Physiology, vol. 289, no. 6, pp. H2334-H2341, 2005.

[51] L. Luo, D. Liu, C. Tang et al., "Sulfur dioxide upregulates the inhibited endogenous hydrogen sulfide pathway in rats with pulmonary hypertension induced by high pulmonary blood flow," Biochemical and Biophysical Research Communications, vol. 433, no. 4, pp. 519-525, 2013.

[52] I. Brüske, R. Hampel, Z. Baumgärtner et al., "Ambient air pollution and lipoprotein-associated phospholipase $\mathrm{A}_{2}$ in survivors of myocardial infarction," Environmental Health Perspectives, vol. 119, no. 7, pp. 921-926, 2011.

[53] V. Lenters, C. S. Uiterwaal, R. Beelen et al., "Long-term exposure to air pollution and vascular damage in young adults," Epidemiology, vol. 21, no. 4, pp. 512-520, 2010.

[54] D. Liu, Z. He, L. Wu, and Y. Fang, "Effects of induction/inhibition of endogenous heme oxygenase-1 on lipid metabolism, endothelial function, and atherosclerosis in rabbits on a high fat diet," Journal of Pharmacological Sciences, vol. 118, no. 1, pp. 14-24, 2012.

[55] Y. Wang, X. Zhao, H. Jin et al., "Role of hydrogen sulfide in the development of atherosclerotic lesions in apolipoprotein E knockout mice," Arteriosclerosis, Thrombosis, and Vascular Biology, vol. 29, no. 2, pp. 173-179, 2009.

[56] T. P. Vacek, S. Rahman, S. Yu, D. Neamtu, S. Givimani, and S. C. Tyagi, "Matrix metalloproteinases in atherosclerosis: role 
of nitric oxide, hydrogen sulfide, homocysteine, and polymorphisms," Vascular Health and Risk Management, vol. 11, pp. 173183, 2015.

[57] Y. Shen, Z. Shen, S. Luo, W. Guo, and Y. Z. Zhu, "The cardioprotective effects of hydrogen sulfide in heart diseases: from molecular mechanisms to therapeutic potential," Oxidative Medicine and Cellular Longevity, vol. 2015, Article ID 925167, 13 pages, 2015.

[58] H. S. Kim, K.-A. Kong, H. Chung, S. Park, and M. H. Kim, "ER stress induces the expression of Jpk, which inhibits cell cycle progression in F9 teratocarcinoma cell," Annals of the New York Academy of Sciences, vol. 1095, pp. 76-81, 2007.

[59] S.-E. Choi, Y.-J. Lee, H.-J. Jang et al., "A chemical chaperone 4-PBA ameliorates palmitate-induced inhibition of glucosestimulated insulin secretion (GSIS)," Archives of Biochemistry and Biophysics, vol. 475, no. 2, pp. 109-114, 2008.

[60] M.-M. Zhao, J.-Y. Yang, X.-B. Wang, C.-S. Tang, J.-B. Du, and H.-F. Jin, "The PI3K/Akt pathway mediates the protection of $\mathrm{SO}_{2}$ preconditioning against myocardial ischemia/reperfusion injury in rats," Acta Pharmacologica Sinica, vol. 34, no. 4, pp. 501-506, 2013.

[61] H. F. Jin, Y. Wang, X. B. Wang, Y. Sun, C. S. Tang, and J. B. Du, "Sulfur dioxide preconditioning increases antioxidative capacity in rat with myocardial ischemia reperfusion (I/R) injury," Nitric Oxide-Biology and Chemistry, vol. 32, pp. 56-61, 2013.

[62] P. Huang, Y. Sun, J. Yang et al., "The ERK1/2 signaling pathway is involved in sulfur dioxide preconditioning-induced protection against cardiac dysfunction in isolated perfused rat heart subjected to myocardial ischemia/reperfusion," International Journal of Molecular Sciences, vol. 14, no. 11, pp. 22190-22201, 2013.

[63] Y. Liang, D. Liu, T. Ochs et al., "Endogenous sulfur dioxide protects against isoproterenol-induced myocardial injury and increases myocardial antioxidant capacity in rats," Laboratory Investigation, vol. 91, no. 1, pp. 12-23, 2011.

[64] A. A. Noronha-Dutra, E. M. Steen-Dutra, and N. Woolf, "Epinephrine-induced cytotoxicity of rat plasma. Its effects on isolated cardiac myocytes," Laboratory Investigation, vol. 59, no. 6, pp. 817-823, 1988.

[65] C. W. Younce and P. E. Kolattukudy, "MCP-1 causes cardiomyoblast death via autophagy resulting from ER stress caused by oxidative stress generated by inducing a novel zinc-finger protein, MCPIP," Biochemical Journal, vol. 426, no. 1, pp. 43-53, 2010.

[66] S. Chen, J. Du, Y. Liang et al., "Sulfur dioxide inhibits excessively activated endoplasmic reticulum stress in rats with myocardial injury," Heart and Vessels, vol. 27, no. 5, pp. 505-516, 2012.

[67] H. Jin, A. D. Liu, L. Holmberg et al., "The role of sulfur dioxide in the regulation of mitochondrion-related cardiomyocyte apoptosis in rats with isopropylarterenol-induced myocardial injury," International Journal of Molecular Sciences, vol. 14, no. 5, pp. 10465-10482, 2013.

[68] S. Chen, J. Du, Y. Liang, R. Zhang, C. Tang, and H. Jin, "Sulfur dioxide restores calcium homeostasis disturbance in rat with isoproterenol-induced myocardial injury," Histology and Histopathology, vol. 27, no. 9, pp. 1219-1226, 2012.

[69] A. Nie and Z. Meng, "Modulation of L-type calcium current in rat cardiac myocytes by sulfur dioxide derivatives," Food and Chemical Toxicology, vol. 44, no. 3, pp. 355-363, 2006.
[70] A. Nie and Z. Meng, "Sulfur dioxide derivative modulation of potassium channels in rat ventricular myocytes," Archives of Biochemistry and Biophysics, vol. 442, no. 2, pp. 187-195, 2005.

[71] X.-B. Wang, H.-F. Jin, C.-S. Tang, and J.-B. Du, “The biological effect of endogenous sulfur dioxide in the cardiovascular system," European Journal of Pharmacology, vol. 670, no. 1, pp. 1-6, 2011.

[72] S.-Y. Chen, H.-F. Jin, Y. Sun, Y. Tian, C.-S. Tang, and J.-B. Du, "Impact of sulfur dioxide on hydrogen sulfide/cystathionine- $\gamma$ lyase and hydrogen sulfide/mercaptopyruvate sulfurtransferase pathways in the pathogenesis of hypoxic pulmonary hypertension in rats," Zhonghua Er Ke Za Zhi, vol. 49, no. 12, pp. 890-894, 2011.

[73] Y. K. Wang, A. J. Ren, X. Q. Yang et al., "Sulfur dioxide relaxes rat aorta by endothelium-dependent and -independent mechanisms," Physiological Research, vol. 58, no. 4, pp. 521-527, 2009.

[74] H.-J. Ma, X.-L. Huang, Y. Liu, and Y.-M. Fan, "Sulfur dioxide attenuates LPS-induced acute lung injury via enhancing polymorphonuclear neutrophil apoptosis," Acta Pharmacologica Sinica, vol. 33, no. 8, pp. 983-990, 2012.

[75] J. L. Hart, "Role of sulfur-containing gaseous substances in the cardiovascular system," Frontiers in Bioscience (Elite Edition), vol. 3, no. 2, pp. 736-749, 2011. 


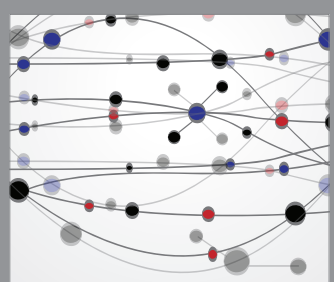

The Scientific World Journal
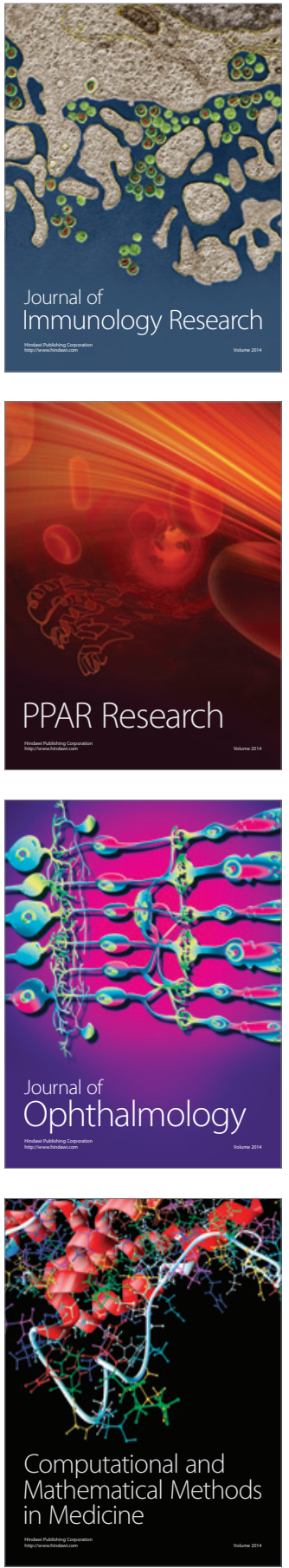

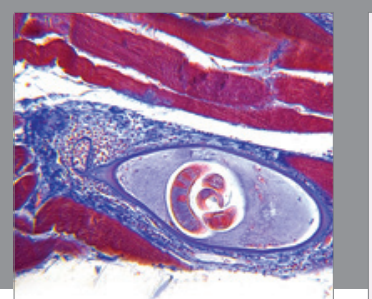

Gastroenterology Research and Practice

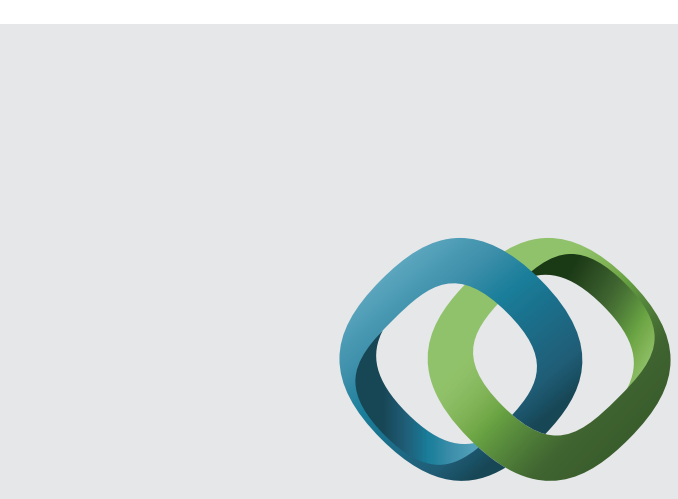

\section{Hindawi}

Submit your manuscripts at

http://www.hindawi.com
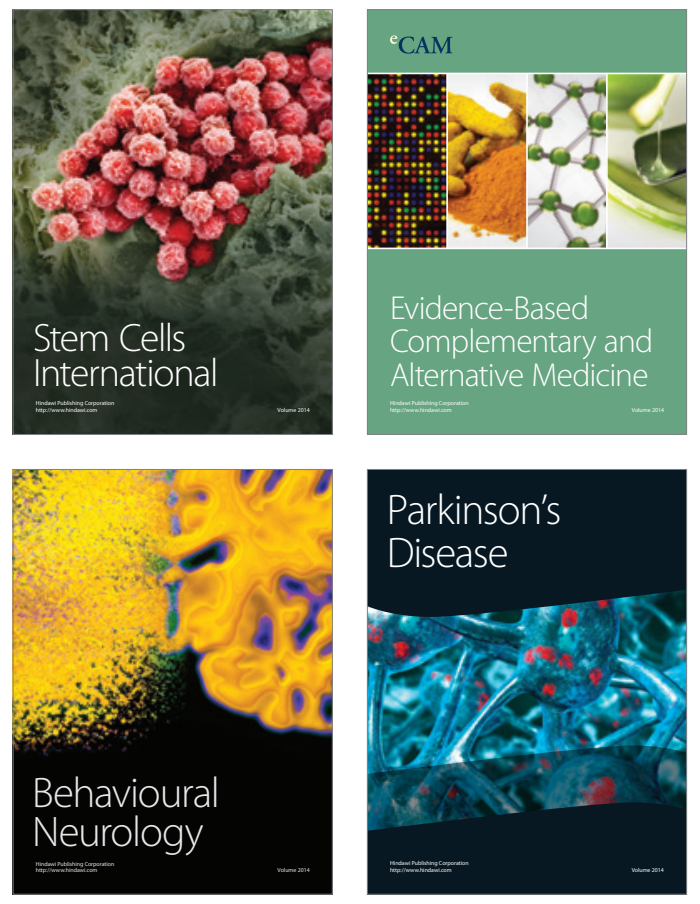
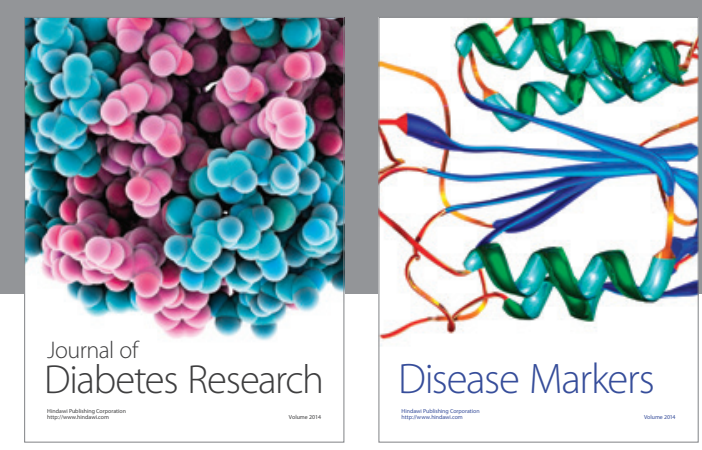

Disease Markers
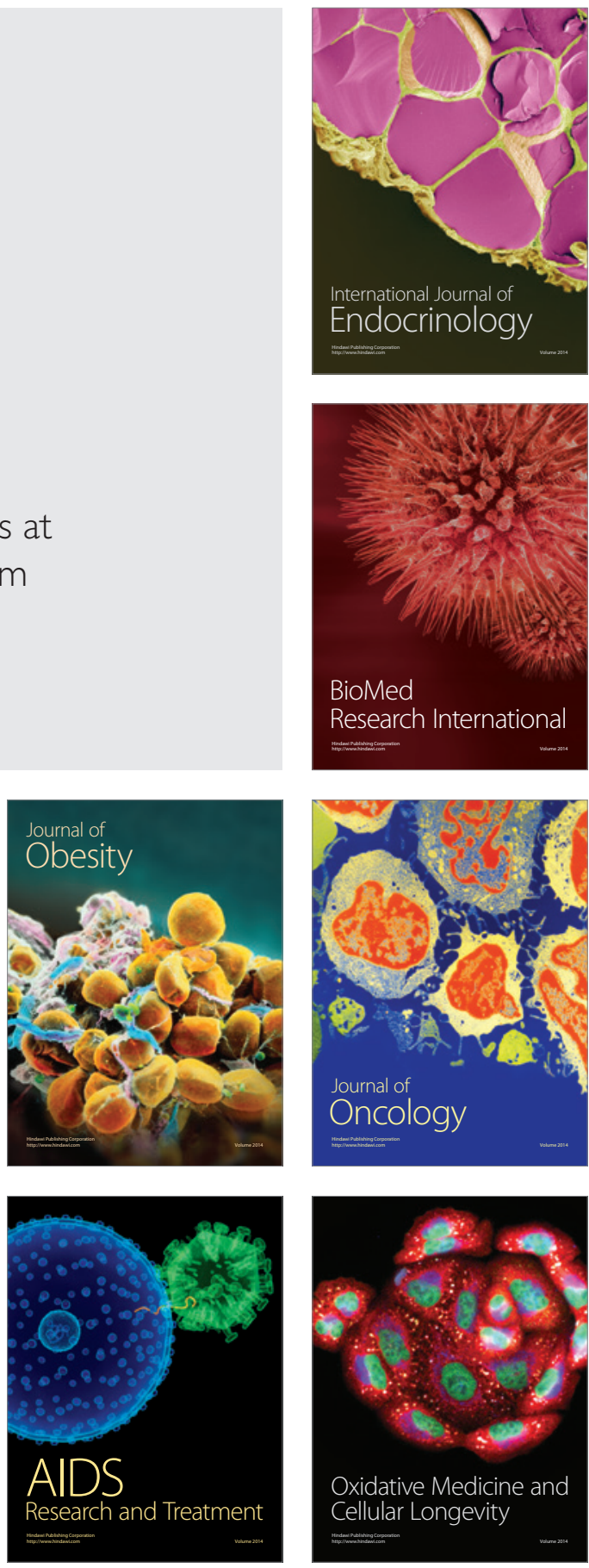